\title{
Performance Analysis of Peer-to-Peer V2V Wireless Communications in the Presence of Interference
}

\author{
Khaled Eshteiwi, Kais Ben Fredj, Georges Kaddoum, and François Gagnon \\ Department of Electrical Engineering, University of Quebec, ÉTS, Montreal, Canada \\ Email: khaled-mohamed.eshteiwi.1@ens.etsmtl.ca,kais@emt.inrs.ca, \{georges.kaddoum, francois.gagnon\}@etsmtl.ca
}

\begin{abstract}
Vehicular Ad-Hoc networks (VANETs) have become an interesting research area especially with growing need to improve the quality of service and the performance of vehicleto-vehicle $(\mathrm{V} 2 \mathrm{~V})$ wireless communication systems. This paper analyses a peer-to-peer $\mathrm{V} 2 \mathrm{~V}$ wireless communication system in the presence of interference. In this vein, the performance metrics of the proposed system are investigated over Nakagami- $m$ fading channels where no interference cancellation is assumed at the receiver side. In particular, the mathematical expression of the probability density function (PDF) of the resultant signal-tointerference-plus-noise ratio (SINR) of the system is derived. The closed-form expression of the PDF derived here lays the foundation for deriving analytical expressions for the moment generating function and the probability of the average bit error rate of the system under consideration. Finally, the closeness of our analytical expressions are corroborated through Monte Carlo simulation results.

Index Terms-V2V communications, Bit error rate, Moment generating function, Probability density function, Nakagamai- $m$ fading channel.
\end{abstract}

\section{INTRODUCTION}

In vehicular communications, $\mathrm{V} 2 \mathrm{~V}$ and vehicle-toinfrastructure (V2I) wireless communication systems are promising techniques to provide reliable and strong communications between users. These types of systems can also be used to extend the coverage area, road safety, and network connectivity. Vehicular wireless communications have received significant attention in recent years due to their unique characteristics that distinguish them from Mobile AdHoc networks (MANETs), including their rapid adjustment to topology changes and their high mobility, enabling them to form a highly dynamic network. The main drawback of VANETs is the network instability, which reduces its efficiency.

$\mathrm{V} 2 \mathrm{~V}$ wireless communications have been limited by the impact of interference, as this latter degrades and bounds the system performance due to the complexity involved in extracting the desired signal. The system performance is especially diminished due to the interferences that occurs when a large number of devices are located close to the useful communication link. In millimeter wave communications, the interference from oncoming vehicles in the opposite lane in Inter-Vehicle Communication (IVC) is presented in [1].

The work in [2] evaluates the impact of co-channel interference in $\mathrm{V} 2 \mathrm{~V}$ communications at $60 \mathrm{GHz}$, with the received signal at the destination vehicle being effected by interference at the same frequency in a highway situation. Due to vehicles mobility, the links quality in $\mathrm{V} 2 \mathrm{~V}$ communications is often decayed due to multipath fading, shadowing and Doppler shift. It is therefore crucial to analyse the impact of interference on $\mathrm{V} 2 \mathrm{~V}$ wireless communications so we can evaluate the performance of such systems in real life situations.

An efficient approach to determine the accuracy of the interference model in wireless networks is proposed in [3], based on the outage probability. An accuracy index closed formula is derived to evaluate the accuracy of the interference model. This method compares two types of interference models, such as the interference ball model (IBM) [4] and the protocol model (PRM) [5].

The authors in [6] have studied the effect of buildings on cellular networks at higher frequencies in urban areas in terms of average data rate, connectivity, and coverage probability. Their results show that there is a positive effect of the buildings signal blockage, as it suppresses the effect of the interference on the useful signal. However, the building blockage can be an obstacle to the transmitted desired signal and interrupt the communication link. A practical measurement of the impact of co-channel interference and the impact of buildings on the performance of $\mathrm{V} 2 \mathrm{~V}$ at urban intersections is presented in [12].

In this paper, we study the key performance metrics of peerto-peer V2V wireless communications system under the impact of interference. The channels are modeled as Nakagami$m$ fading channels, which are versatile enough for many situations, and are independent but not necessarily identical. The main contribution of this work is the derivation of a new mathematical expression of the PDF for the total SINR. A special case of PDF for Rayleigh fading channels is also presented. Consequently, the moment generating function (MGF) expression for the proposed system is obtained based on the aforementioned PDF. A closed-form expression for the average bit error rate (ABER) for $\mathrm{V} 2 \mathrm{~V}$ communication under the impact of interference is also derived. To fill the research gap in the vehicular communications domain and to the best of the authors knowledge, there is no previous work touching the analytical analysis for such a model.

The rest of this paper is organized as follows. Section II presents the system and the channel models. The performance analysis is provided in Section III. In Section IV, numerical, simulation results and comments are presented and discussed before concluding the manuscript in Section V. 


\section{SySTEM MODEL}

The system model considered in this paper includes four nodes: two source vehicles $S_{1}$ and $S_{2}$, two destination vehicles $D_{1}$ and $D_{2}$. Each pair of vehicles prefer to communicate directly, such that $S_{1}$ communicates with $D_{1}$ and $S_{2}$ communicates with $D_{2}$ as shown in Fig. 1. Also, the signals from $S_{1}$ to $D_{2}$ and from $S_{2}$ to $D_{1}$ are treated as interference signals at each destination $D_{1}$ and $D_{2}$ respectively. Therefore, there are two received signals at each node $D_{1}$ and $D_{2}$. At $D_{1}$, the first signal is from node $S_{1}$ and represents the desired signal, and the second signal is from the node $S_{2}$ and is considered as an interference signal. At $D_{2}$, the first signal is from node $S_{2}$ and represents the useful signal, and the second signal is from the node $S_{1}$ and is treated as an interference signal. The parameters $h_{S_{1} D_{1}}, h_{S_{2} D_{2}}, h_{S_{1} D_{2}}$ and $h_{S_{2} D_{1}}$ are the channel coefficients in the links $S_{1} D_{1}$, $S_{2} D_{2}, S_{1} D_{2}$ and $S_{2} D_{1}$ respectively, modelled as Nakagami$m$ fading. The channels are mutually independent and nonidentically distributed (i.n.i.d) with zero mean and variance $\sigma_{i}^{2}$ where $i \in\left\{S_{1} D_{1}, S_{2} D_{2}, S_{1} D_{2}, S_{2} D_{1}\right\}$.

For simplicity, we assume $D \in\left\{D_{1}, D_{2}\right\}, S \in\left\{S_{1}, S_{2}\right\}$, $S D \in\left\{S_{1} D_{1}, S_{2} D_{2}\right\}, I D \in\left\{S_{2} D_{1}, S_{1} D_{2}\right\}$ and $I \in$ $\left\{S_{2}, S_{1}\right\}$. Due to the symmetry of the channels and equidistant between each two vehicles, the received signals at each $D$ is given by

$$
y_{D}=\sqrt{E_{S}} h_{S D} x_{S}+\sqrt{E_{I}} h_{I D} x_{I}+n_{S D},
$$

where $x_{S} \in\{+1,-1\}$ and $x_{I}$ are the transmitted BPSK symbols at $S$ and $I, E_{S}$ and $E_{I}$ are the transmitted signal energies at $S$ and $I$ respectively, and $n_{S D}$ is white Gaussian noise (AWGN) in the $S D$ channel with variance $\sigma_{S D}^{2}$. According to Eq. (1), the received SINR $\gamma_{D}$ can be easily found as

$$
\gamma_{D}=\frac{\gamma_{S D}}{\gamma_{I D}+1}
$$

where $\gamma_{S D}$ and $\gamma_{I D}$ are the SNR of $S D$ and $I D$ links at the destination node respectively.

\section{Performance Analysis}

In this section, we present the analytical expression derivations used to compute the PDF, MGF and ABER for SINR in $\mathrm{V} 2 \mathrm{~V}$ wireless communications.

\section{A. Probability Density Function}

The channel coefficients in the proposed system are modeled as Nakagami $-m$ distribution, and, therefore, $\left|h_{i j}\right|^{2}$ follows gamma distribution. Since the channels are i.n.i.d, the PDF of $h_{S D}$ and $h_{I D}$ channel gain coefficients can be respectively written as [13]

$$
\begin{aligned}
f_{\gamma_{S D}}(x) & =\frac{C_{S D}}{\Gamma\left(m_{S D}\right)} x^{m_{S D}-1} \exp \left(-x C_{S D}\right), \\
f_{\gamma_{I D}}(x) & =\frac{C_{I D}}{\Gamma\left(m_{I D}\right)} x^{m_{I D}-1} \exp \left(-x C_{I D}\right),
\end{aligned}
$$

where $\Gamma(z)$ denotes the gamma function and is given by $\Gamma(z)=\int_{0}^{\infty} \exp (-t) t^{z-1} d t\left[7\right.$, Eq. (8.310.1)], $m_{i}>0.5$

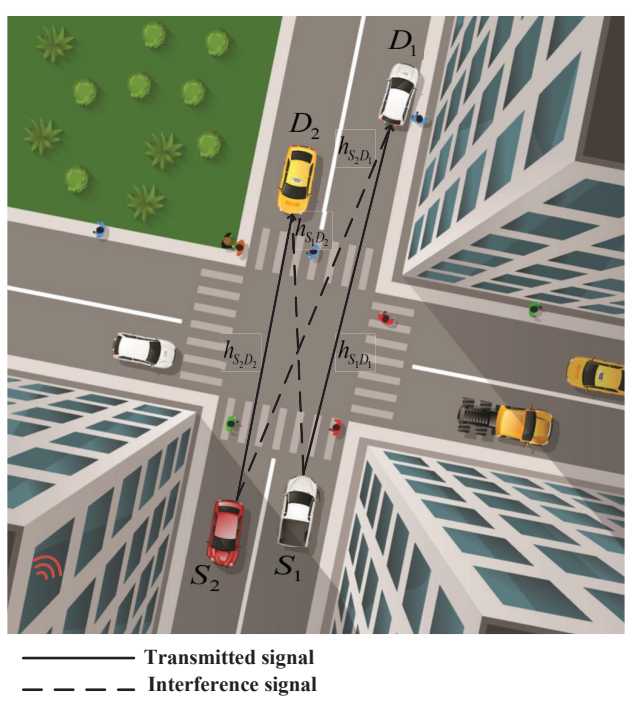

Fig. 1: System model.

is the shape parameter, $C_{i}=\frac{m_{i}}{\bar{\gamma}_{i}}, \gamma_{i}=h_{i}^{2} E_{S} / N_{o}$ is the instantaneous SNR, $\bar{\gamma}_{i}=\mathbb{E}\left(\left|h_{i}\right|^{2}\right) E_{S} / N_{o}$ is the average SNR and $\mathbb{E}($.$) is the expectation operator. The total SINR at D$ can be found by using the convolution of the PDFs of $\gamma_{S D}$ and $\gamma_{I D}$. Let $\gamma_{S D}$ and $\gamma_{I D}$ be independent random variables with PDFs $f_{\gamma_{S D}}\left(\gamma_{S D}\right)$ and $f_{\gamma_{I D}}\left(\gamma_{I D}\right)$ respectively. Then the CDF of $\gamma_{D}$ can be calculated as [14]

$$
\begin{aligned}
F_{\gamma_{D}}(x)= & P\left(\frac{\gamma_{S D}}{\gamma_{I D}+1} \leq x\right) \\
=P & \left(\gamma_{S D} \geq x\left(\gamma_{I D}+1\right) / \gamma_{I D}+1<0\right) \\
& \quad+P\left(\gamma_{S D} \leq x\left(\gamma_{I D}+1\right) / \gamma_{I D}+1>0\right)
\end{aligned}
$$

We can rewrite Eq. (5) in integral form as

$$
\begin{aligned}
F_{\gamma_{D}}(x) & =\int_{-\infty}^{-1}\left[\int_{x\left(\gamma_{I D}+1\right)}^{\infty} f_{\gamma_{S D}}\left(\gamma_{S D}\right) d \gamma_{S D}\right] f_{\gamma_{I D}}\left(\gamma_{I D}\right) d \gamma_{I D} \\
& +\int_{-1}^{\infty}\left[\int_{-\infty}^{x\left(\gamma_{I D}+1\right)} f_{\gamma_{S D}}\left(\gamma_{S D}\right) d \gamma_{S D}\right] f_{\gamma_{I D}}\left(\gamma_{I D}\right) d \gamma_{I D}
\end{aligned}
$$

In order to drive the PDF of $\gamma_{D}$, we need to differentiate Eq. (6). Also, it should be noted that the first term in the right hand side is ignored because it represents the negative part and the PDF is always non-negative everywhere. After the differentiation and some mathematical manipulations, we get

$$
\begin{aligned}
f_{\gamma_{D}}(x) & =\frac{d}{d x} F_{\gamma_{D}}(x) \\
= & \int_{0}^{\infty}\left(\gamma_{I D}+1\right) f_{\gamma_{S D}, \gamma_{I D}}\left(x\left(\gamma_{I D}+1\right), \gamma_{I D}\right) d \gamma_{I D}
\end{aligned}
$$

where $f_{A, B}(a, b)$ is a joint probability distribution function of two independent random variables $a$ and $b$. 
Using $t=\gamma_{I D}+1$, Eq. (7) becomes

$$
f_{\gamma_{D}}(x)=\int_{1}^{\infty} t f_{\gamma_{S D}}(x t) f_{\gamma_{I D}}(t-1) d t .
$$

Substituting Eq. (3) and Eq. (4) into Eq. (8), the function $f_{\gamma_{D}}(x)$ can be written as

$$
\begin{array}{r}
f_{\gamma_{D}}(x)=\frac{C_{S D}^{m_{S D}} C_{I D}^{m_{I D}} x^{m_{S D}-1}}{\Gamma\left(m_{S D}\right) \Gamma\left(m_{I D}\right)} \int_{1}^{\infty} t^{m_{S D}}(t-1)^{m_{I D}} \\
\quad \times \exp \left(-x t C_{S D}\right) \exp \left(-(t-1) C_{I D}\right) d t
\end{array}
$$

Changing the integral limits and putting $\tau=t-1$, Eq. (9) can be expressed as

$$
\begin{aligned}
& f_{\gamma_{D}}(x)=\frac{C_{S D}^{m_{S D}} C_{I D}^{m_{I D}} x^{m_{S D}-1} \exp \left(-x C_{S D}\right)}{\Gamma\left(m_{S D}\right) \Gamma\left(m_{I D}\right)} \\
& \times \sum_{j=0}^{m_{S D}}\left(\begin{array}{c}
m_{S D} \\
j
\end{array}\right) \int_{0}^{\infty} \tau^{m_{I D}+j-1} \exp \left(-\left(C_{I D}+x C_{S D}\right) \tau\right) d \tau .
\end{aligned}
$$

After some algebraic manipulations, the closed-form expression for the total PDF at $D$ can be obtained in closed form as

$$
\begin{aligned}
f_{\gamma_{D}}(x)=\frac{C_{S D}^{m_{S D}} C_{I D}^{m_{I D}} x^{m_{S D}-1} \exp \left(-x C_{S D}\right)}{\Gamma\left(m_{S D}\right) \Gamma\left(m_{I D}\right)} \\
\quad \times \sum_{j=0}^{m_{S D}}\left(\begin{array}{c}
m_{S D} \\
j
\end{array}\right) \frac{\Gamma\left(m_{I D}+j\right)}{\left(x C_{S D}+C_{I D}\right)^{m_{I D}+j}},
\end{aligned}
$$

where $\left(\begin{array}{l}n \\ k\end{array}\right)=\frac{n !}{k !(n-k) !}$ is the binomial coefficient. For the special case where the fading parameters of the desired and interference signals are equal, i.e $m_{S D}=m_{I D}=1$, which represents Rayleigh fading distribution, the PDF expression in Eq. (11) simplifies to

$$
f_{\gamma_{D}}(x)=\frac{\exp \left(-x / \bar{\gamma}_{S D}\right)}{\bar{\gamma}_{S D} \bar{\gamma}_{I D}}\left[\frac{\frac{x}{\bar{\gamma}_{S D}}+\frac{1}{\bar{\gamma}_{I D}+}+1}{\left(\frac{x}{\bar{\gamma}_{S D}}+\frac{1}{\bar{\gamma}_{I D}}\right)^{2}}\right],
$$

where $\bar{\gamma}_{S D}$ is the average SNR of the $S-D$ channel and $\bar{\gamma}_{I D}$ is the average SNR of the $I-D$ channel.

\section{B. Moment Generating Function}

In this subsection, we derive the MGF expression based on the PDF of the total SINR provided in Eq. (11). The MGF will be used to determine the ABER for our system model. The MGF definition is given as [8]

$$
M_{\gamma_{D}}(s)=\int_{0}^{\infty} \exp (-s x) f_{\gamma_{D}}(x) d x .
$$

Inserting Eq. (11) into Eq. (13), the MGF can be written as

$$
\begin{aligned}
& M_{\gamma_{D}}(s)=\int_{0}^{\infty} \exp (-s x) \frac{C_{S D}^{m_{S D}} C_{I D}^{m_{I D}} x^{m_{S D}-1}}{\Gamma\left(m_{S D}\right) \Gamma\left(m_{I D}\right)} \\
& \times \exp \left(-x C_{S D}\right) \sum_{j=0}^{m_{S D}}\left(\begin{array}{c}
m_{S D} \\
j
\end{array}\right) \frac{\Gamma\left(m_{I D}+j\right)}{\left(x C_{S D}+C_{I D}\right)^{m_{I D}+j}} d x .
\end{aligned}
$$

By simplifying Eq. (14), we obtain

$$
\begin{aligned}
& M_{\gamma_{D}}(s)= \\
& \quad \frac{C_{S D}^{m_{S D}} C_{I D}^{m_{I D}} x^{m_{S D}-1}}{\Gamma\left(m_{S D}\right) \Gamma\left(m_{I D}\right)} \sum_{j=0}^{m_{S D}}\left(\begin{array}{c}
m_{S D} \\
j
\end{array}\right) \Gamma\left(m_{I D}+j\right) \\
& \quad \times \int_{0}^{\infty} x^{m_{S D}-1} \frac{\exp \left(-\left(s+C_{S D}\right) x\right)}{\left(x C_{S D}+C_{I D}\right)^{m_{I D}+j}} d x .
\end{aligned}
$$

Comparing Eq. (15) to [9, Eq. (2.3.6.9)] and after some mathematical manipulations, the MGF of $\gamma_{D}$ becomes

$$
\begin{aligned}
& M_{\gamma_{D}}(s)=\frac{C_{S D}^{m_{S D}} C_{I D}^{m_{I D}}}{\Gamma\left(m_{I D}\right)} \sum_{j=0}^{m_{S D}}\left(\begin{array}{c}
m_{S D} \\
j
\end{array}\right) \\
& \quad \times \Gamma\left(m_{I D}+j\right)\left(\frac{C_{I D}}{C_{S D}}\right)^{m_{S D}-m_{I D}-j} \frac{1}{\left(C_{S D}\right)^{m_{I D}+j}} \\
& \times \Psi\left(m_{S D}, m_{S D}+1-m_{I D}-j ;\left(C_{S D}+s\right)\left(\frac{C_{I D}}{C_{S D}}\right)\right),
\end{aligned}
$$

where $\Psi(., . ;$.$) is the Tricomi confluent hypergeometric func-$ tion of the second kind, given by [7]

$$
\begin{array}{r}
\Psi(a, b ; z)=\frac{1}{\Gamma(a)} \int_{0}^{\infty} e^{-z t} t^{a-1}(1+t)^{b-a-1} d t, \\
{[\operatorname{Re}(a)>0, \operatorname{Re}(z)>0] .}
\end{array}
$$

\section{Average Bit Error Rate}

In this subsection, the probability of error based on the MGF obtained in Eq. (16) is derived. The average error probability depends on the type of the modulation used in the proposed system. The error probability can be written as [10, Eq. (8.22)]

$$
P(e)=\frac{1}{\pi} \int_{0}^{\beta} M_{\gamma_{D}}\left(\frac{\alpha_{M P S K}}{\sin ^{2} \beta}\right) d \beta,
$$

where $\alpha_{M P S K}=\sin ^{2}(\pi / M), \beta=(M-1) \pi / M$ and $M_{\gamma_{D}}($. is the moment generating function. Substituting the MGF formula obtained in Eq. (16) into Eq. (18) is intractable to find the ABER. Therefore, the ABER for the BPSK modulation can be obtained as [11, Eq.(14)] in approximate closed form, and be given by

$$
\begin{aligned}
P(e)= & \left(\frac{\beta}{2 \pi}\right) M_{\gamma_{D}}\left(\alpha_{M P S K}\right)+\left(\frac{4 \beta-2 \pi}{8 \pi}\right) \\
& \times M_{\gamma_{D}}\left(\frac{\alpha_{M P S K}}{\sin ^{2} \beta}\right)+\frac{1}{4} M_{\gamma_{D}}\left(\frac{4 \alpha_{M P S K}}{3}\right),
\end{aligned}
$$

where the expression $M_{\gamma_{D}}$ is as given in Eq. (16). 


\section{Simulation Results}

In this section, we present the numerical results of the analytical PDF, MGF and ABER of V2V wireless networks where BPSK modulation is considered. The system includes the effect of the interference over independent but not necessarily identically distributed Nakagami- $m$ fading channels. Without loss of generality, the channels $h_{S D}$ and $h_{I D}$ are assumed to be zero-mean complex Gaussian random variables with equal variance $\sigma_{i}^{2}=1$.

For all forthcoming results, we have used the Monte Carlo simulation to generate $10^{6}$ samples and the other corresponding case-specific system parameters are included in the caption of each figure. The simulation results are provided to illuminate and verify the analytical results. We plot a comparison between the analytical and the simulated PDF that also represent ratio of two Nakagami- $m$ fading channel distributions in Fig. 2. The results obtained with different simulation confirm a perfect agreement between two curves with different values of $m_{S D}$ and $m_{I D}$ at $\gamma_{S D}=2 \mathrm{~dB}$ and $\gamma_{I D}=-3 \mathrm{~dB}$.

Fig. 3 shows different presentations of the PDF of the SNR for several values of $\gamma_{I D}$ at $m_{S D}=3, m_{I D}=2$ and $\gamma_{S D}=4$ $\mathrm{dB}$. It should be noted that the maximum value of the PDF occurs at low values of abscissa. It is obvious that higher values of $f_{\gamma_{D}}(x)$ can be reached at higher $\gamma_{I D}$ rates.

Fig. 4 shows an excellent match between simulation and theoretical results of the MGF of $\mathrm{V} 2 \mathrm{~V}$ communication in the presence of interference under dissimilar values of $m$ - fading parameters $m_{S D}$ and $m_{I D}$. We can observe that higher values of $M_{\gamma_{D}}(x)$ are achieved at lower abscissa when $\gamma_{S D}=2 \mathrm{~dB}$ and $\gamma_{I D}=-3 \mathrm{~dB}$. Fig. 5 shows the accuracy of the matching for the mathematical derivation and the analytical results. We can see that the MGF achieves higher values when $\gamma_{I D}$ is high.

Fig. 6 shows the performance of the proposed system in terms of the ABER compared to the average SNR in an approximated expression. When the fading parameter of the source-destination channel increases, the ABER also decreases. It is clear that at low and medium SNR, the analytical approximation is close enough to the simulation. Fig. 7 represents different curves of the ABER versus the SNR for the cases of $\gamma_{I D}=-5,0$ and $5 \mathrm{~dB}$. Also, the fading parameter $m_{S D}=3$ and $m_{I D}=2$ are used for the $S-D$ and $I$ $D$ channels respectively. It is also observed that increasing $\gamma_{I D}$ will augment the overall ABER and degrade the system performance as expected. In general, Fig. 6 and Fig. 7 reveal that the ABER achieves better performance at high SNR values.

\section{CONClusions}

The impact of interference on the performance of $\mathrm{V} 2 \mathrm{~V}$ wireless communications is studied and analysed over independent and non-identically distributed Nakagami- $m$ fading channels. The closed-form expression of the PDF of the total SINR is derived for peer-to-peer $\mathrm{V} 2 \mathrm{~V}$ communications under the effect of interference, an aspect that represents the novelty of

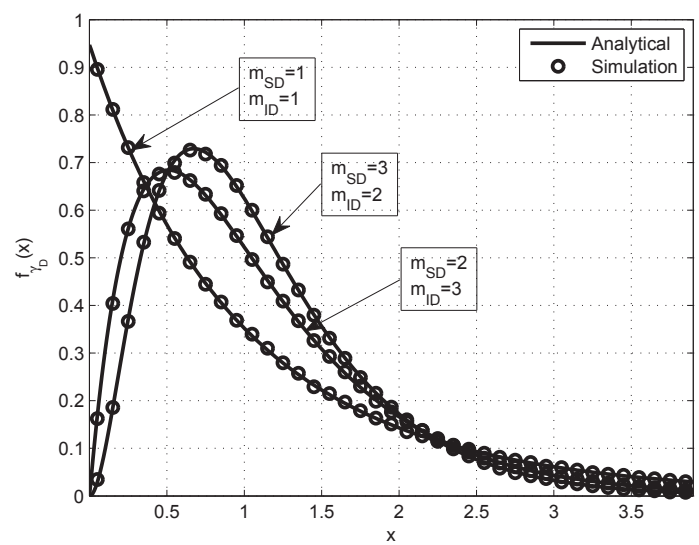

Fig. 2: Analytical and simulation results for the PDF of SNR of $\mathrm{V} 2 \mathrm{~V}$ wireless communications in the presence of interference over Nakagami- $m$ fading channels for different values of $m_{S D}$ and $m_{I D}$ with $\gamma_{S D}=2 \mathrm{~dB}$ and $\gamma_{I D}=-3 \mathrm{~dB}$.

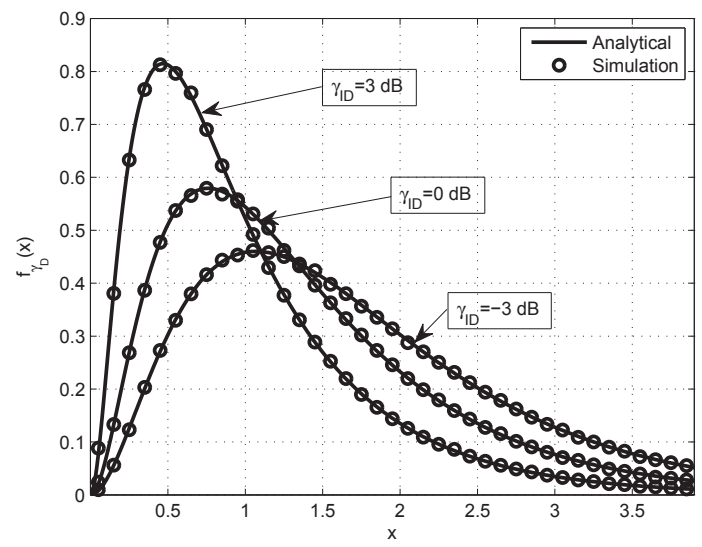

Fig. 3: Analytical and simulation results for the PDF of SNR of $\mathrm{V} 2 \mathrm{~V}$ wireless communications in the presence of interference over Nakagami- $m$ fading channels for different values of $\gamma_{I D}, \gamma_{S D}=4$ $\mathrm{dB}, m_{S D}=3$ and $m_{I D}=2$.

this work. The PDF is then used to obtain the mathematical closed-form expression of the MGF of the proposed system. The ABER of the system under consideration is derived based on the MGF, in an approximated form. Monte Carlo simulation results confirm the accuracy of the obtained expressions. This paper concentrates on the impact of interference on V2V communications and provides essential mathematical expressions such as the PDF, MGF and ABER in particular. This will help researchers in the field to design full duplex relaying systems and predict its behavior with respect to channel parameters, including self-interference.

\section{REFERENCES}

[1] L. B. Michael, "Interference characteristics in inter-vehicle communication from oncoming vehicles," in Proc. IEEE VTC, Sep. 1999, pp. 753- 


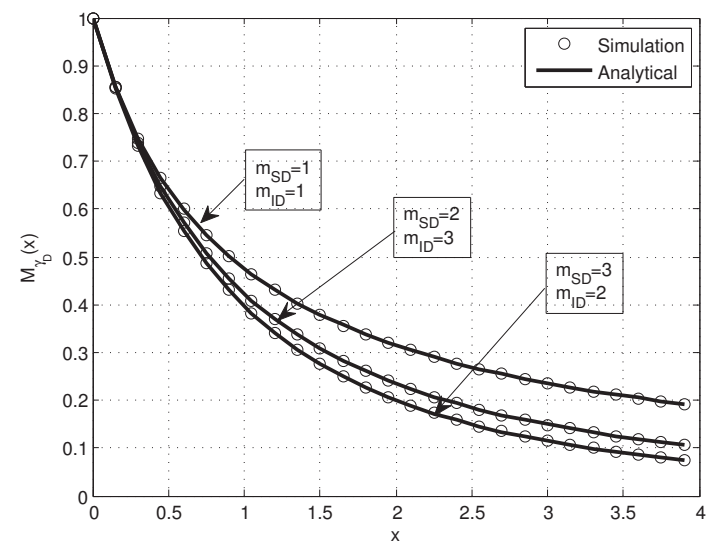

Fig. 4: Analytical and simulation results for the MGF of SNR of V2V wireless communications including interference over Nakagami$m$ fading channels for several values of $m_{S D}$ and $m_{I D}$ with $\gamma_{S D}=2$ $\mathrm{dB}$ and $\gamma_{I D}=-3 \mathrm{~dB}$.

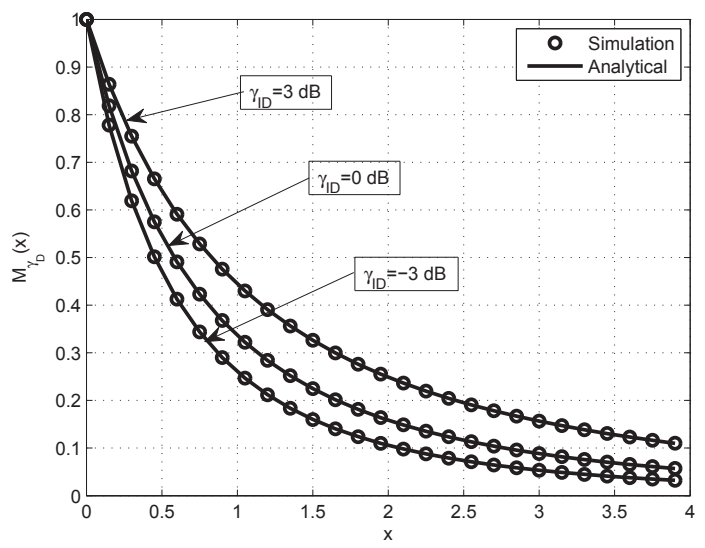

Fig. 5: Analytical and simulation results for the MGF of SNR of $\mathrm{V} 2 \mathrm{~V}$ wireless communications including interference over Nakagami$m$ fading channels for $m_{S D}=3, m_{I D}=2, \gamma_{S D}=2 \mathrm{~dB}$ and different values of $\gamma_{I D}$.

757

[2] O. Andrisano, M. Chiani, V. Tralli, and R. Verdone, "Impact of cochannel interference on vehicle-to-vehicle communications at millimeter waves," in IEEE Int. Conf. Commun. Syst. ICC, Singapore, Nov. 16-20, 1992.

[3] H. Shokri-Ghadikolaei, C. Fischione, and E. Modiano, "On the accuracy of interference models in wireless communications," in Proc. IEEE Int. Conf. Commun. (ICC), Kuala Lumpur, 2016, pp. 1-6.

[4] L. B. Le, E. Modiano, C. Joo, and N. B. Shroff, "Longest-queuefirst scheduling under sinr interference model," in Proc. of the eleventh ACM Int. sympos. on Mobile ad hoc networking and computing. ACM, 2010, pp. 41-50.

[5] P. Gupta and P. R. Kumar, "The capacity of wireless networks," IEEE Trans. inf. theory, vol. 46, no. 2, pp. 388-404, 2000.

[6] T. Bai, R. Vaze, and R. W. Heath, "Analysis of blockage effects on urban cellular networks," IEEE Trans. Wireless Commun., vol. 13, no. 9, pp. 5070-5083, 2014.

[7] A. Jeffrey and D. Zwillinger, Table of integrals, series, and products. Academic Press, 2007.

[8] J. Malhotra, "Performance analysis of diversity combining multichannel receivers in generic-gamma fading channels," Tamkang Journal of Science

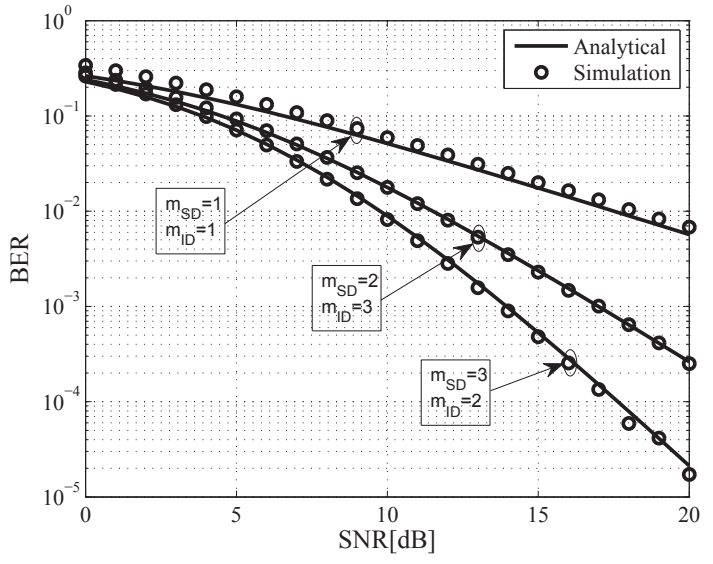

Fig. 6: Analytical and simulation results for the BER of SNR of $\mathrm{V} 2 \mathrm{~V}$ wireless communications with different values of $m_{S D}, m_{I D}$ and $\gamma_{I D}=-5 \mathrm{~dB}$

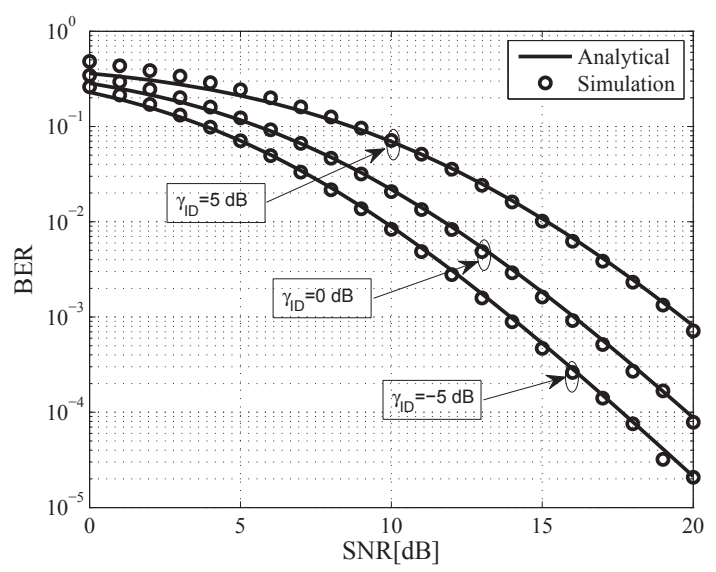

Fig. 7: Analytical and simulation results for the BER of SNR of $\mathrm{V} 2 \mathrm{~V}$ wireless communications for $m_{S D}=3, m_{I D}=2$ and different values of $\gamma_{I D}$.

and Engineering, vol. 14, no. 4, pp. 333-340, 2011

[9] A. Prudnikov, Yu. Brychkov and Oleg Marichev, Integrals and series. Vol. 1. AAPT, 1988.

[10] M. K. Simon and M.-S. Alouini, Digital communication over fading channels. Vol. 95, John Wiley and Sons, 2005.

[11] A. H. Forghani, S. S. Ikki, and S. Aissa, "On the performance and power optimization of multihop multibranch relaying networks with cochannel interferers," IEEE Trans. Vehicular Technology, vol. 62, no. 7, pp. 34373443, 2013.

[12] Tchouankem, Hugues and Lorenzen, Torsten, "Measurement-based evaluation of interference in Vehicular Ad-Hoc Networks at urban intersections, “ IEEE Int. Conf. , Commun. Workshop (ICCW), pp. 2381-2386, 2015.

[13] Imene Trigui, Sofiene Affes, and Alex Stephenne, "Closed-form error analysis of dual-hop relaying systems over Nakagami- $m$ fading channels, "IEEE Wireless Commun. and Networking Conf. (WCNC), pp. 1-5, 2010.

[14] A. Papoulis and S. U. Pillai, "Probability, Random Variables, and Stochastic Processes,“. McGraw-Hill, 2001 